\title{
Unit of Magnetic Flux
}

National Cancer Institute

\section{Source}

National Cancer Institute. Unit of Magnetic Flux. NCI Thesaurus. Code C68792.

A unit of measurement of the quantity of magnetism, being designated as the total

number of magnetic lines of force passing through a specified area in a magnetic field. 